\title{
Evaluation of left ventricular systolic function with pulsed wave tissue Doppler in rheumatic mitral stenosis
}

\author{
Saadet Guven ${ }^{1}$, Taner Sen ${ }^{2}$, Omac Tufekcioglu \\ Esra Gucuk $^{3}$, Belma Uygur ${ }^{4}$, Erkan Kahraman ${ }^{5}$ \\ ${ }^{1}$ Department of Cardiology, Yozgat Sorgun State Hospital, Turkey \\ ${ }^{2}$ Department of Cardiology, Dumlupinar University Kutahya Evliya \\ Celebi Education and Research Hospital, Turkey \\ ${ }^{3}$ Department of Cardiology, Turkiye Yuksek Ihtisas Hospital, Turkey \\ ${ }^{4}$ Department of Cardiology, Karaman State Hospital, Turkey \\ ${ }^{5}$ Department of Cardiology, Tekirdag State Hospital, Turkey
}

\begin{abstract}
Background: Mitral stenosis (MS) is still the most common complication of acute rheumatic fever in Turkey. Rheumatic carditis affects not only cardiac valves but also myocardium. In this study, we aimed to evaluate the subclinical left ventricular (LV) systolic dysfunction and contraction of short and long axial circumferential and longitudinal fibers by pulsed wave tissue Doppler in rheumatic MS patients who have preserved LV systolic function in $2 D$ echocardiography.
\end{abstract}

Methods: Fifteen severe, 20 moderate rheumatic MS patients hospitalized for mitral balloon valvuloplasty, and 15 patients who had normal echocardiographic findings were included in the study. After routine conventional transthoracic echocardiographic examination, LV myocardial systolic velocities were evaluated with pulsed wave tissue Doppler in the short and long axis with simultaneous electrocardiographic monitoring.

Results: Long axis first systolic velocity (SW1) of mild-moderate and severe MS was much lower than normal group $(10.7 \pm 2.3$ in normal group vs. $7.9 \pm 1.3$ in mild-moderate $M S$ group vs. $6.2 \pm 1.4$ in severe MS group, $p<0.001)$. Long axis $Q-S W 1$ duration was longer in mild-moderate $M S$ group (145 \pm 32 in normal group vs. $199 \pm 43$ in mild-moderate $M S$ group, $p=0.001$ ). Short axis $Q-S W 2$ duration was longer in normal group compared to mild-moderate and severe MS groups (298 \pm 41 in normal group vs. $245 \pm 37$ in mild-moderate MS group vs. $234 \pm 26$ in severe MS group, $p<0.001)$. Significant correlation between mitral valve area and $S W 1, Q-S W 1$ was determined $(p=0.01)$.

Conclusions: Even if LV functions are normal with conventional $2 D$ echocardiography, subclinical systolic dysfunction exists in MS. Also, there is a dyssynchrony between contraction of longitudinal and circumferential myofibrils. (Cardiol J 2014; 21, 1: 33-38)

Key words: mitral stenosis, pulsed wave tissue Doppler

Address for correspondence: Taner Sen, MD, Dumlupinar University Kutahya Evliya Celebi Education and Research Hospital Cardiology Department, Turkey, e-mail: medicineman_tr@hotmail.com

Received: 01.03.2013

Accepted: 02.05.2013 


\section{Introduction}

Mitral stenosis (MS) is still the most common complication of acute rheumatic fever in Turkey. Carditis affects not only cardiac valves but also myocardium. Conventional echocardiographic methods give valuable information about valvular involvement and function but they are not enough for the evaluation of myocardial involvement. In previous studies the authors showed that patients with MS had significantly impaired long-axis function evaluated by M-mode or tissue Doppler echocardiography despite normal global systolic function [1-3].

In this study, we aimed to evaluate the subclinical left ventricular (LV) systolic dysfunction and contraction of short and long axial circumferential and longitudinal fibers by pulsed wave tissue Doppler in rheumatic MS patients who preserved $\mathrm{LV}$ systolic function in 2D echocardiography.

\section{Methods}

\section{The characteristic of the study}

Thirty-five patients who had moderate to severe rheumatic MS hospitalized for mitral balloon valvuloplasty in Turkiye Yuksek Ihtisas Education and Research Hospital between September 2010 and January 2011 were included as the study group. Fifteen patients who had normal echocardiographic findings and similar characteristics with study group were selected as the control group. All participants were informed about the study and their consents were obtained.

\section{Exclusion criteria}

Exclusion criteria are given below:

- atrial fibrillation, atrial flutter and other tachy-brady arrhytmias;

- electrocardiographic bundle branch block;

- preexcitation syndromes;

- coronary artery disease;

- non rheumatic valve disease;

- moderate to severe valvular regurgitation.

\section{Echocardiographic examination}

All patients underwent transthoracic echocardiographic examination by Vivid pro 7 (GE Horten, Norway) echocardiography device with a 1.5-3.6 MHz transducer. All echocardiographic examinations were performed by the same operator who was blinded for groups of the patients. LV systolic diameter (LVSD) and LV diastolic diameter (LVDD), left atrium diameters and mitral valve area (MVA) with pressure half time and planimetric methods were noted. LV ejection fraction (LVEF) with biplane area method from apical window and fractional shortening (FS) with M-mode echocardiography according to the formula: FS $(\%)=$ $=([\mathrm{LVDD}-\mathrm{LVSD}] / \mathrm{LVDD} \times 100)$ were calculated.

LV myocardial systolic velocities were examined with pulsed wave tissue Doppler (PWTD) in the short and long axis with simultaneous electrocardiographic (ECG) monitoring. Apical and parasternal windows were used for PWTD echocardiography. To measure velocities along the short axis, the sample volume was positioned on the mid-portion of the middle region of the posterior LV wall on a parasternal long-axis view and velocities along the long axis were measured by putting the sample volume in the subendocardial portion of the middle region of the posterior $\mathrm{LV}$ wall on an apical long-axis view. Each PWTD measurement revealed two peak systolic velocities (SW1 - first systolic velocity peak; SW2 - second systolic velocity peak). Also, the durations from $\mathrm{Q}$ wave in ECG to SW1 and SW2 were noted respectively as Q-SW1 and Q-SW2 (Fig. 1). The average of 3 cycles was calculated.

\section{Statistical analysis}

All data were evaluated by the SPSS (Statistical for Social Sciences for Windows, version 17.0). Parametric and nonparametric distribution of the variables was assessed by Kolmogorov-Smirnov test. Parametric data were expressed as mean \pm standard deviation and qualitative data as numbers and percentages. Pearson test was used for correlation analysis of the parametric data. One-way ANOVA test was used for the comparison of the quantitative data between the groups. The results were considered statistically significant at the level of $\mathrm{p}<0.05$.

\section{Results}

Fifty patients included in the study were divided into 3 groups according to severity of MS as normal mitral valve, mild-moderate MS $\left(\mathrm{MVA}>1.1 \mathrm{~cm}^{2}\right)$ and severe MS $\left(\mathrm{MVA} \leq 1.1 \mathrm{~cm}^{2}\right)$.

Basal characteristics were similar between the groups (Table 1). There were no statistical differences in heart rate, systolic and diastolic blood pressure, EF, FS, LVDD and LVSD between the groups. Left atrium diameter and systolic pulmonary artery pressure were not different between the groups evaluated with one-way ANOVA test (Table 2).

Long axis SW1 velocities of the mild-moderate and the severe MS were much lower than the nor- 


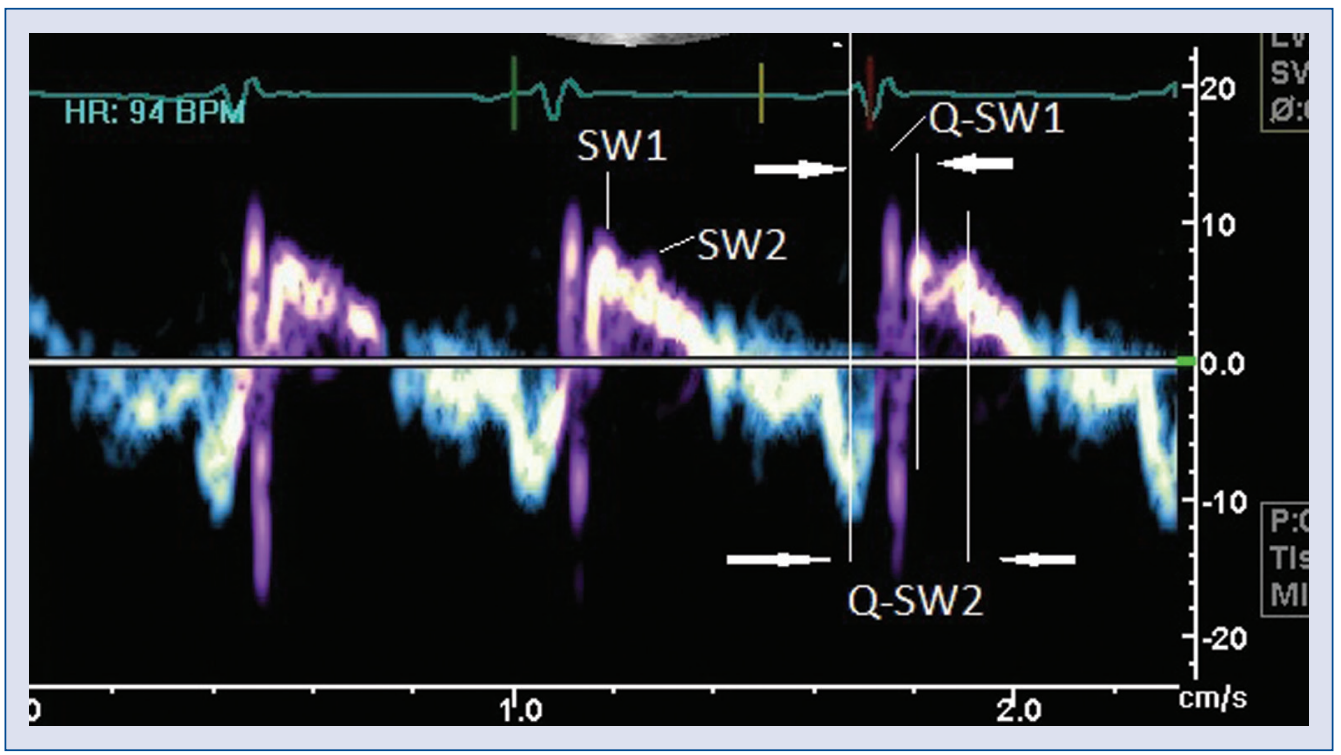

Figure 1. Pulsed wave tissue Doppler velocities of left ventricle posterior wall in the apical long axis view; SW1 — first systolic velocity peak and SW2 - second systolic velocity peak. Also, the duration from Q wave in ECG to SW1 and SW2 were noted respectively as Q-SW1 and Q-SW2.

Table 1. Characteristic features of the patients.

\begin{tabular}{lccc}
\hline & $\begin{array}{c}\text { Normal group } \\
(\mathbf{n}=\mathbf{1 5})\end{array}$ & $\begin{array}{c}\text { Mild-moderate MS } \\
(\mathbf{n = 2 0 )}\end{array}$ & $\begin{array}{c}\text { Severe MS } \\
\text { (n= 15) }\end{array}$ \\
\hline Age [year] & $36 \pm 8$ & $44 \pm 10$ & $42 \pm 10$ \\
Sex (female/male) & $11 / 4$ & $17 / 3$ & $13 / 2$ \\
Heart rate [bpm] & $77 \pm 11$ & $76 \pm 13$ & $78 \pm 8$ \\
Systolic blood pressure [mm Hg] & $112 \pm 12$ & $114 \pm 16$ & $106 \pm 12$ \\
Diastolic blood pressure [mm Hg] & $73 \pm 7$ & $71 \pm 10$ & $68 \pm 9$ \\
Acetylsalicylic acid & 0 & $16(80 \%)$ & $12(80 \%)$ \\
Beta-blocker & $1(6.6 \%)$ & $15(75 \%)$ & $11(73 \%)$ \\
Diuretic & 0 & $5(25 \%)$ & $12(80 \%)$ \\
Calcium channel blocker & 0 & 0 & $1(6.6 \%)$ \\
Digoxin & 0 & $1(5 \%)$ & 0 \\
\hline
\end{tabular}

MS - mitral stenosis

Table 2. Echocardiographic findings.

\begin{tabular}{lccc}
\hline & $\begin{array}{c}\text { Normal group } \\
(\mathbf{n}=\mathbf{1 5})\end{array}$ & $\begin{array}{c}\text { Mild-moderate MS } \\
\text { (n= 20) }\end{array}$ & $\begin{array}{c}\text { Severe MS } \\
\text { (n= 15) }\end{array}$ \\
\hline Mitral valve area [mm²] & $>40$ & $14 \pm 1.7$ & $10 \pm 0.8$ \\
Mean mitral gradient [mm Hg] & - & $6.8 \pm 3$ & $10.8 \pm 2.1$ \\
Interventricular septum [mm] & $8.4 \pm 0.7^{* *}$ & $9.6 \pm 0.9$ & $9.4 \pm 1.1$ \\
LVPW [mm] & $8.6 \pm 0.6$ & $9.6 \pm 0.94$ & $9.0 \pm 1.2$ \\
Left atrial diameter [mm] & $33 \pm 2^{*}$ & $44 \pm 3$ & $46 \pm 5$ \\
LVDD [mm] & $28 \pm 3$ & $29 \pm 4$ & $28 \pm 3$ \\
LVSD [mm] & $44 \pm 2$ & $46 \pm 3$ & $43 \pm 10$ \\
Fractional shortening [\%] & $35 \pm 3$ & $35 \pm 4$ & $36 \pm 4$ \\
Ejection fraction [\%] & $65 \pm 3$ & $65 \pm 6$ & $65 \pm 5$ \\
SPAP [mm Hg] & $23 \pm 2 \#, \&$ & $36 \pm 12 \$$ & $52 \pm 13$ \\
\hline
\end{tabular}

${ }^{*} \mathrm{p}<0.001$ compared to mild-moderate and severe MS; ${ }^{*} \mathrm{p}<0.05$ compared to mild-moderate and severe MS; \#p $<0.01$ compared to mild-moderate MS; \&p < 0.001 compared to severe MS; $\$ p<0.001$ compared to severe MS; MS — mitral stenosis; LVPW — left ventricular posterior wall; LVDD - left ventricular diastolic diameter; LVSD — left venticular systolic diameter; SPAP — systolic pulmonary artery pressure 
Table 3. Pulsed wave tissue Doppler recordings.

\begin{tabular}{lccc}
\hline & Normal group $(\mathbf{n}=\mathbf{1 5})$ & Mild-moderate MS $(\mathbf{n}=\mathbf{2 0})$ & Severe MS $(\mathbf{n}=\mathbf{1 5})$ \\
\hline Long axis & $10.7 \pm 2.3^{*}$ & $7.9 \pm 1.3$ & $6.2 \pm 1.4$ \\
SW1 [cm/s] & $5.4 \pm 0.7$ & $6.0 \pm 1.2^{* *}$ & $4.7 \pm 1.2$ \\
SW2 [cm/s] & $145 \pm 32^{* * *}$ & $199 \pm 43$ & $180 \pm 46$ \\
Q-SW1 [ms] & $259 \pm 37$ & $258 \pm 43$ & $258 \pm 44$ \\
Q-SW2 [ms] & $7.5 \pm 1.2$ & & $7.0 \pm 1.4$ \\
Short axis & $4.8 \pm 1.0$ & $7.1 \pm 1.2$ & $5.5 \pm 1.3$ \\
SW1 $[\mathrm{cm} / \mathrm{s}]$ & $182 \pm 28$ & $5.5 \pm 1.1$ & $168 \pm 28$ \\
SW2 $[\mathrm{cm} / \mathrm{s}]$ & $298 \pm 41^{*}$ & $178 \pm 38$ & $234 \pm 26$ \\
Q-SW1 [ms] & $245 \pm 37$ & \\
Q-SW2 [ms] & & & \\
\hline
\end{tabular}

${ }^{*} p<0.001$ compared to mild-moderate and severe MS; ${ }^{* *} p<0.01$ compared to severe MS; ${ }^{* *} p=0.001$ compared to mild-moderate MS; MS - mitral stenosis; SW1 - first systolic velocity peak; SW2 - second systolic velocity peak; Q-SW1 - the duration from $\mathrm{Q}$ wave to SW1; $\mathrm{Q}-\mathrm{SW} 2$ - the duration from $\mathrm{Q}$ wave to SW2

mal group $(10.7 \pm 2.3$ in normal group vs. $7.9 \pm$ \pm 1.3 in mild-moderate MS group vs. $6.2 \pm 1.4$ in severe MS group, $\mathrm{p}<0.001$ ). Long axis SW2 velocities were found statistically different between mild-moderate and severe MS (6.0 \pm 1.2 vs. $4.7 \pm$ $\pm 1.2, \mathrm{p}<0.01)$. Long axis Q-SW1 duration was longer in mild-moderate MS group $(145 \pm 32$ in normal group vs. $199 \pm 43$ in mild-moderate MS group, $\mathrm{p}=0.001$ ). Short axis SW1, SW2 and Q-SW1 mean values were not different between the groups but short axis Q-SW2 duration was longer in the normal group compared to the mild-moderate and the severe MS groups $(298 \pm 41$ in normal group vs. $245 \pm 37$ in mild-moderate MS group vs. $234 \pm 26$ in severe MS group, $\mathrm{p}<0.001$ ) (Table 3 ). Correlation between MVA and SW1, Q-SW1 was investigated with Pearson test. Significant correlation between MVA and SW1, Q-SW1was determined $(\mathrm{p}=0.01)($ Table 4$)$.

\section{Discussion}

Histological studies showed that LV myocardium was composed of circumferential fiber in the middle portion, longitudinal fibers in subendocardial and subepicardial portions [4, 5]. LV systolic function is accomplished by contraction of both fibers [6]. Longitudinal fibers contract earlier in early systole and LV takes a shape of a sphere during isovolumetric contraction phase [7-9]. Immediately after this, circumferential fibers contract and LVEF is completed $[10,11]$.

In previous studies, contraction of circumferential and longitudinal fibers was investigated with conventional M-mode echocardiography [12]. With the help of new modalities, myocardial tissue velocities could be noninvasively measured $[13,14]$.
Table 4. The correlation between mitral valve area and pulsed wave tissue Doppler parameters.

\begin{tabular}{lcc}
\hline & $\begin{array}{c}\text { Pearson } \\
\text { correlation }\end{array}$ & $\begin{array}{c}\text { Sig. } \\
\text { (2-tailed) }\end{array}$ \\
\hline SW1 long axis & $-0.431^{*}$ & 0.002 \\
SW2 long axis & 0.226 & 0.115 \\
Q-SW1 long axis & $0.483^{*}$ & 0.000 \\
Q-SW2 long axis & -0.008 & 0.955 \\
SW1 short axis & -0.116 & 0.423 \\
SW2 short axis & 0.228 & 0.111 \\
Q-SW1 short axis & -0.036 & 0.803 \\
Q-SW2 short axis & $-0.471^{*}$ & 0.001 \\
\hline
\end{tabular}

${ }^{*}$ Correlation significance $\mathrm{p}<0.01$ (2-tailed); SW1 - first systolic velocity peak; SW2 - second systolic velocity peak; Q-SW1 - the duration from $\mathrm{Q}$ wave to SW 1 ; Q-SW 2 - the duration from $\mathrm{Q}$ wave to SW2

It would be possible to get valuable information about LV short and long axial myocardial functions with tissue Doppler echocardiography [15-19].

It is generally assumed that LV functions are preserved in pure MS. However, varying degrees of LV systolic dysfunction were detected in previous studies [20,21]. Many hemodynamic and myocardial factors have been blamed for LV dysfunction in MS, such as reduction in LV filling, chronic myocardial inflammation, scarring of subvalvular apparatus, reduction of LV compliance and diastolic dysfunction, increased afterload, abnormal right-left septal interaction, and pulmonary hypertension [22]. It still remains a challenge whether LV systolic dysfunction is a result of functional or myocardial factors in patients with MS.

When we look at the earliest studies, in 1953, Grant [23] in his article suggested that part of the 
LV was atrophic in patients with MS. He found that the posterior wall of the LV in patients with MS was shortened and atrophic. These ventricular changes were explained to be due to partial immobilization of the ventricle by the fibrotic posterior mitral leaflet and also to the chronic ventricular inactivity resulting from long-standing reduced ventricular filling. In 1968, Feigenbaum et al. [24] showed that significant increase in LV end diastolic pressure occurred despite increase in MVA in patients with MS who had had mitral valvulotomy.

The concept of ventricular atrophy would also explain this increase in LV end diastolic pressure because of abnormal compliance of the LV. We can also derive from these studies that chronic volume underload of the $\mathrm{LV}$ due to mitral valve stenosis over years and decades may be responsible for $\mathrm{LV}$ systolic dysfunction.

Lee et al. [25] demonstrated that MS patients with reduced global LV function usually had segmental contraction abnormalities. In their study, they also showed that most of MS patients who had impaired $\mathrm{EF}$ gained improvement in LV function after mitral valvuloplasty, while some did not. They concluded that both myocardial and mechanical factors would be responsible for LV dysfunction in patients with MS [25].

Acute rheumatic carditis affects not only cardiac valves but also myocardial tissue. In our study, we evaluated the subclinical LV systolic dysfunction and contraction of short and long axial circumferential and longitudinal fibers by PWTD in rheumatic MS patients who preserved LV systolic function in 2D echocardiography. Contraction of longitudinal fibers in isovolumetric contraction phase is impaired in MS patients compared to normal healthy subjects as a result mean longitudinal axis SW1 velocity decrease and this decrease is correlated with the severity of MS ( $p<0.001)$. Moreover, the time required for stimulation of longitudinal fibers increases and this increase is correlated with the severity of MS $(p=0.01)$. As a result, while LV functions are normal with conventional 2D echocardiography in MS, subclinical systolic dysfunction is present and there is also a dyssynchrony between circumferential and longitudinal myofibrils.

In their study, Ozdemir et al. [20] showed the myocardial velocities of the LV indicating $\mathrm{LV}$ function were found to be significantly lower in patients with pure MS. In the study performed by Ozer at al. [21] it was showed that patients with MS had significantly impaired long-axis function evaluated by M-mode or tissue Doppler echocardiography despite normal global systolic function.
In their study, Arat et al. [26] found that myocardial $\mathrm{S}$ and $\mathrm{E}$ velocities and the $\mathrm{E} / \mathrm{A}$ ratio were increased after percutaneous mitral valve balloon valvotomy with no significant change in the LVEF and the total ejection isovolume index which is more reflective of overall cardiac function than systolic or diastolic functions alone. They emphasized that a subclinical LV dysfunction might be responsible for no significant change in LVEF.

Inflammation and edema due to acute rheumatic fever resulting in fusion, thickening and shortening of mitral and subvalvular apparatus also affects anatomical and contractile features of myofibrils [27-29]. In their ultrastructural study, Lee at al. [25] showed intense myofibril loss due to both myofibril degeneration and impaired mitochondria to myofibril ratio in pure rheumatic MS patients. As a result they specified that the main pathological mechanism responsible for $\mathrm{LV}$ function disturbance was myocardial involvement due to rheumatic process in rheumatic pure MS [30]. Colle et al. [31] revealed that rheumatic MS patients had anterior and posterior hypokinesia and mean shortening of hypokinetic segments was lower in MS patients than in normal individuals. They also showed that patients who had contractile abnormality had lower EF and circumferential myofibril shortening velocity.

\section{Conclusions}

In our study, we showed that mild-moderate and severe MS patients had significantly lower myocardial systolic velocity which was a marker of quantitative contractile function than normal subjects despite the fact that they had similar EF values. We still do not exactly know that which factors are more important (myocardial, mechanical or both) in case of LV dysfunction in MS patients. However, we exactly know that LV functions are not preserved and at least a subclinical LV dysfunction exists in pure MS.

\section{Conflict of interest: none declared}

\section{References}

1. Tufekcioglu O, Aras D, Yildiz A, Topaloglu S, Maden O. Myocardial contraction properties along the long and short axes of the left ventricle in isolated left ventricular non-compaction: Pulsed tissue Doppler echocardiography. Eur J Echocardiography, 2008; 9: 344-350.

2. Oki T, Tabata T, Mishiro Y, Yamada H et al. Pulsed tissue Doppler imaging of left ventricular systolic and diastolic wall motion velocities to evaluate differences between long and short axes in healthy subjects. J Am Soc Echocardiogr, 1999; 12: 308-313. 
3. Mishiro Y, Oki T, Yamada H, Wakatsuki T, Ito S. Evaluation of left ventricular contraction abnormalities in patients with dilated cardiomyopathy with the use of pulsed tissue Doppler imaging. J Am Soc Echocardiogr, 1999; 12: 913-920.

4. Grant RP. Notes on the muscular architecture of the left ventricle. Circulation, 1965; 32: 301-308.

5. Greenbaum RA, Ho SY, Gibson DG, Becker AE, Anderson RH. Left ventricular fibre architecture in man. Br Heart J, 1981; 45: 248-263.

6. Rademakers FE, Rogers WJ, Guir WH et al. Relation of regional cross-fiber shortening to wall thickening in the intact heart: Three-dimensional strain analysis by NMR tagging. Circulation, 1994; 89: 1174-1182.

7. Ichida F, Hamamichi Y, Miyawaki $\mathrm{T}$ et al. Clinical features $\mathrm{f}$ isolated noncompaction of the ventricular myocardium: Long-term clinical course, hemodynamic properties, and genetic background. J Am Coll Cardiol, 1999; 34: 233-240.

8. Freedom RM, Yoo SJ, Perrin D, Taylor G, Petersen S, Anderson RH. The morphological spectrum of ventricular noncompaction. Cardiol Young, 2005; 15: 345-364.

9. Hamamichi Y, Ichida F, Hashimoto I et al. Isolated noncompaction of the ventricular myocardium: Ultrafast computed tomography and magnetic resonance imaging. Int J Cardiovasc Imag, 2001; 17: 305-314.

10. Jenni R, Goebel N, Tartini R, Schneider J, Arbenz U, Oelz O. Persisting myocardial sinusoids of both ventricles as an isolated anomaly: Echocardiographic, angiographic, and pathologic anatomical findings. Cardiovasc Intervent Radiol, 1986; 9: 127-131.

11. Oechslin EN, Attenhofer Jost CH, Rojas JR, Kaufmann PA, Jenni R. Long-term follow-up of 34 adults with isolated left ventricular noncompaction: A distinct cardiomyopathy with poor prognosis. J Am Coll Cardiol, 2000; 36: 493-500.

12. Ichida F, Hamamichi Y, Miyawaki T et al. Clinical features $\mathrm{f}$ isolated noncompaction of the ventricular myocardium: Long-term clinical course, hemodynamic properties, and genetic background. J Am Coll Cardiol, 1999; 34: 233-240.

13. Yamazaki N, Mine Y, Sano A et al. Analysis of ventricular wall motion using color-coded tissue Doppler imaging system. Jpn J Appl Physiol, 1994; 33: 3141-3146.

14. Garcia MJ, Rodriguez L, Ares M et al. Myocardial wall velocity assessment by pulsed Doppler tissue imaging: Characteristic findings in normal subjects. Am Heart J, 1996; 132: 648-656.

15. Oki T, Tabata T, Yamada $\mathrm{H}$ et al. Clinical application of pulsed Doppler tissue imaging for assessing abnormal left ventricular relaxation. Am J Cardiol, 1997; 79: 921-928.

16. Yamada H, Oki T, Tabata T, Iuchi A, Ito S. Assessment of left ventricular systolic wall motion velocity with pulsed tissue Doppler imaging: Comparison with peak $\mathrm{dP} / \mathrm{dt}$ of the left ventricular pressure curve. J Am Soc Echocardiogr, 1998; 11: 442-449.

17. Garcia MJ, Rodriguez L, Ares M, Griffin BP, Thomas JD, Klein AL. Differentiation of constrictive pericarditis from restrictive cardio- myopathy: Assessment of left ventricular diastolic velocities in longitudinal axis by Doppler tissue imaging. J Am Coll Cardiol, 1996; 27: 108-114.

18. Nagueh SF, Middleton KJ, Kopelen HA, Zoghbi WA, Quinones MA. Doppler tissue imaging: A noninvasive technique for evaluation of left ventricular relaxation and estimation of filling pressures. J Am Coll Cardiol, 1997; 30: 1527-1533.

19. Tufekcioglu O, Aras D, Ozeke O, Maden O, Topaloglu S. Comparison of regional systolic myocardial velocities in patients with isolated left ventricular noncompaction and patients with idiopathic dilated cardiomyopathy. J Am Soc Echocardiogr, 2006; 19: 1320-1325.

20. Ozdemir K, Altunkeser BB, Gök H, Içli A, Temizhan A. Analysis of the myocardial velocities in patients with mitral stenosis. J Am Soc Echocardiogr, 2002; 15: 1472-1478.

21. Ozer N, Can I, Atalar E et al. Left ventricular long-axis function is reduced in patients with rheumatic mitral stenosis. Echocardiography, 2004; 21: 107-112.

22. Klein AJ, Carroll JD. Left ventricular dysfunction and mitral stenosis. Heart Fail Clin, 2006; 2: 443-452.

23. Grant RP. Architectonics of the heart. Am Heart J, 1953; 46: 405.

24. Feigenbaum H, Linback RE, Nasser WK. Hemodynamic studies before and after insturemental mitral commissurotomy. A reappraisal of the patophysiology of mitral stenosisand the efficacy of mitral valvotomy. Circulation, 1968; 38: 261-276.

25. Lee TM, Su SF, Chen MF, Liau CS, Lee YT. Changes of left ventricular function after percutaneous balloon mitral valvuloplasty in mitral stenosis with impaired left ventricular performance. Int J Cardiol, 1996; 56: 211-215.

26. Arat N, Yildirim N, Guray U, Tufekcioglu O, Korkmaz S, Sabah I. Evaluation of the global systolic and diastolic function of the left ventricle by the total ejection isovolume index following percutaneous mitral balon valvuloplasty: A tissue Doppler imaging study. Türk Kardiyol Dern Arş, 2006; 34: 10-15.

27. Gash AK, Carabello BA, Cepin D. Left ventriculer ejection performance and systolic muscle function in patient with mitral stenosis. Circulation, 1983; 67:148.

28. Chandrashekhar Y, Westaby S, Narula J. Mitral stenosis. Lancet, 2009; 374: 1271-1283.

29. Carabello BA. Modern management of mitral stenosis. Circulation, 2005; 112: 432-437.

30. Lee YS, Lee CP. Ultrastructural pathological study of left ventricular myocardium in patients with isolated rheumatic mitral stenosis with normal or abnormal left ventricular function. Jpn Heart J, 1990; 31: 435-448.

31. Colle JP, Rahal S, Ohayon J et al. Global left ventricular function and regional wall motion in pure mitral stenosis. Clin Cardiol, 1984; 7: 573-580. 